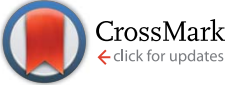

Cite this: RSC Adv., 2014, 4, 62525
Received 1st October 2014 Accepted 30th October 2014

DOI: 10.1039/c4ra11611a

www.rsc.org/advances

\section{Effect of two facile synthetic strategies with alterable polymerization sequence on the performance of $\mathrm{N}$-vinyl carbazole-based conjugated porous materials $\uparrow$}

\author{
Wei Huang, ${ }^{\text {ab }}$ Chuantao Gu, ${ }^{\text {ab }}$ Ting Wang, ${ }^{a}$ Chunyang Gu, ${ }^{\text {ab }}$ Shanlin Qiao*a \\ and Renqiang Yang*a
}

Four ( $N$-vinyl carbazole)-based polymers were prepared through two facile synthetic strategies including both free radical polymerization and oxidative polymerization in different sequences. $\mathrm{N}$-vinyl carbazole containing an ethylenic bond and a carbazole group is a superior candidate to study the effects of polymerization methods and sequences on the performance of conjugated microporous materials (CMPs). P2 was obtained with a BET of $878.46 \mathrm{~m}^{2} \mathrm{~g}^{-1}, 12.8$ times that of $\mathrm{P} 1\left(68.65 \mathrm{~m}^{2} \mathrm{~g}^{-1}\right)$ in path 1 , whereas in path 2, P3 $\left(621.18 \mathrm{~m}^{2} \mathrm{~g}^{-1}\right)$ and P4 $\left(660.62 \mathrm{~m}^{2} \mathrm{~g}^{-1}\right)$ were obtained without obvious difference in surface areas and pore structure. The dominant pore of linear polymer P1 centered at $3.94 \mathrm{~nm}$, which is consistent with the polymers of intrinsic microporous (PIMs) analogues, and the counterparts P2 (ultramicropores, $0.54 \mathrm{~nm}), \mathrm{P} 3(1.13 \mathrm{~nm})$, and P4 $(1.17 \mathrm{~nm})$ are typical CMPs. P2 shows the best gas uptake abilities and absorbancies for organic solvents among the polymers. The results demonstrate that polymerization methods and sequences can have a great influence on the performance of materials, and only by careful choice of polymerization method and fine adjustment of the polymerization sequence can one obtain conjugated porous materials with optimum performance. Path 1 , which is cost effective, high yielding and pollution free, should be regarded as the optimum selection process to prepare the $N$ vinyl carbazole-based porous materials. The reusability of P2 and P4 shows their impressive stability after 5 cycles of use or acid/base treatment. It is worth noting that P2 and P4 with a high porosity and pore volume are promising materials in carbon dioxide uptake, and in methane and hydrogen storage.

\section{Introduction}

In recent years, microporous organic polymers (MOPs) have emerged as an important branch of advanced porous materials, showing great potential in energy-related areas including gas storage and separation and in heterogeneous catalysis. ${ }^{1-6}$ Compared to inorganic or inorganic-organic hybrid microporous materials, MOPs have intrinsic advantages, such as high stability, synthetic diversity and ultrahigh surface areas. MOPs can be separated into two branches, crystalline and amorphous polymers, by the crystal phase. Covalent organic frameworks (COFs) $)^{7,8}$ are the crystalline polymers in MOP materials, while

${ }^{a}$ CAS Key Laboratory of Bio-based Materials, Qingdao Institute of Bioenergy and Bioprocess Technology, Chinese Academy of Sciences, Qingdao 266101, China. E-mail: yangrq@qibebt.ac.cn; Fax: +86 532 80662778; Tel: +86 53280662700

${ }^{b}$ University of Chinese Academy of Sciences, Beijing 100049, China

$\dagger$ Electronic supplementary information (ESI) available: Detailed NMR $\left({ }^{1} \mathrm{H}\right.$ NMR and ${ }^{13} \mathrm{C}$ NMR) of materials Fig. S1 and S2; XRD for P1 to P4 (Fig. S3), additional gas adsorption properties Fig. S4 and S5, selectivity of $\mathrm{CO}_{2} / \mathrm{CH}_{4}$ (Fig. S6). See DOI: $10.1039 / \mathrm{c} 4 \mathrm{ra11611a}$ the amorphous polymers include polymers of intrinsic microporous (PIMs), ${ }^{\mathbf{9}, 10}$ hyper-crosslinked polymers (HCPs) ${ }^{\mathbf{1 1}}$ and conjugated microporous polymers (CMPs). ${ }^{12-15}$ CMPs combining $\pi$-conjugation with permanent microporosity have demonstrated a striking potential in wide applications such as gas uptake, selective adsorption, heterogeneous and other photoelectric related fields. Typically, the CMPs also have advantages such as low mass density, high chemical stability and numerous selectable monomers and synthetic methods due to their organic character. It has been reported that the porous properties of CMPs depend mainly on the length of the rigid connecting strut, topological structure design and synthetic technique. Up to now, most researches have focused on the design of new building blocks and topological investigations. Few studies have explored in detail the effect of synthesis methods and sequences on the CMPs. Typically, there are substantially four methods available to synthesize CMPs, such as the synthesis of CMP-1 to CMP-4 through SonogashiraHagihara cross reactions, ${ }^{13} \mathrm{P} 1$ and $\mathrm{P} 2$ through Suzuki coupling reactions, ${ }^{16}$ TPE-CMP through Yamamoto coupling reactions, ${ }^{17}$ and CPOP-1, P-1 and P-2 by $\mathrm{FeCl}_{3}$ oxidative coupling 
polymerization. ${ }^{\mathbf{1 8 , 1 9}}$ In the first three methods, heavy metals such as palladium or nickel, which are expensive and contaminative, are used to trigger the reaction, so taking this into consideration, the relatively non-polluting and cheap $\mathrm{FeCl}_{3}$ is adopted as the main catalyst in our work.

The unique structure of the $N$-vinyl carbazole-containing ethylenic double bond and carbazole group is a superior candidate with which to study the synthesis methods and sequences effects on CMPs as there are two potential active sites. It is well-known that the $N$-substituted carbazole is a highly electro-active group with low oxidative potential which enables the effective coupling reaction to occur between its oxidative species (radical carbazyl cation) and produces only dimers..$^{20}$ As the 3- and 6-positions of carbazole are oxidation sites, $N$-vinyl carbazole is a potential building block to construct conjugated microporous materials through $\mathrm{FeCl}_{3}$ oxidative coupling. ${ }^{18}$ As to the ethylenic double bond, free radical polymerization is employed to prepare the conventional poly $(N$-vinyl carbazole) (PVK), ${ }^{21}$ and its synthesis technology has reached the level of industrial production. It is worth pointing out that the source material $N$-vinyl carbazole is abundant and can be synthesized easily; meanwhile, the synthesis strategies can be carried out environmentally friendly without a toxic metal catalyst, and have high yield. These advantages make it possible to produce CMPs on a large scale and place practical application on the agenda. In addition, the low framework density, large pore volume and nitrogen-containing nature of the polymers make them candidates as adsorbents for $\mathrm{CO}_{2}$ capture and $\mathrm{H}_{2} / \mathrm{CH}_{4}$ storage.

Here, we designed two facile synthetic paths to prepare poly( $N$-vinyl carbazole) which can fully utilize the two important active sites of the structure with different sequences. In the first step of path 1 and path 2, free radical polymerization and oxidative polymerization are applied to give P1 and P3, where the ethylenic double bond and 3, 6-position in the carbazole group are connected to form backbones, respectively. And through the second step in path 1 and path 2, we aim to polymerize P1 and P3 further by utilizing their important remaining active sites (the 3, 6-position of carbazole in P1 and the ethylenic bond in P3). Thus, $\mathrm{P} 2$ and $\mathrm{P} 4$ were obtained from the same two polymerization methods but in different sequences. The detailed schematic is shown in Scheme 1. Finally, the effects of synthetic methods and sequences on the performance of porous materials were investigated.

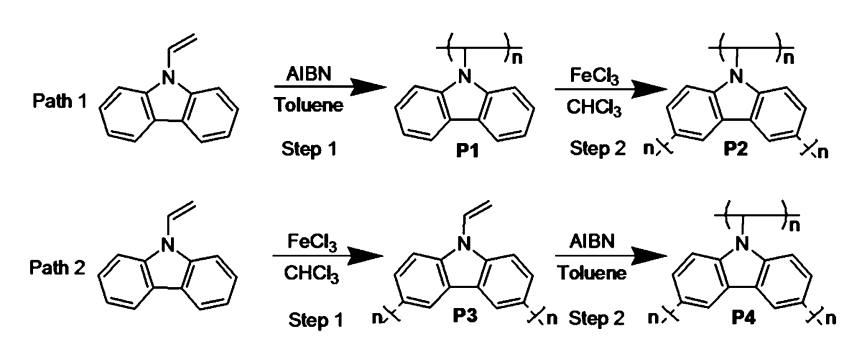

Scheme 1 Synthetic routes of path 1 and path 2 towards the polymers P1 to P4.

\section{Experimental section}

\section{Materials}

Toluene, tetrahydrofuran (THF) and chloroform were purified by distillation prior to use. 2,2'-Azobis-isobutyronitrile (AIBN) and $N$-vinyl carbazole $(\mathrm{VCz})$ were purchased from J\&K. Ferric chloride was obtained from Aldrich. All the reagents were used as received.

\section{Synthesis}

P1: A typical procedure ${ }^{22}$ to prepare poly( $N$-vinyl carbazole) (PVK) was as follows: $\mathrm{VCz}$ (600 mg, $3.1 \mathrm{mmol}$ ) and AIBN (25 mg, 0.155 $\mathrm{mmol}$ ) were dissolved in dry toluene. The mixture was heated to $70{ }^{\circ} \mathrm{C}$ and stirred for $6 \mathrm{~h}$ under a nitrogen atmosphere. Then 100 $\mathrm{mL}$ methanol was added to precipitate the PVK. The resulting precipitate was collected by filtration, washed with methanol three times and dried under vacuum at room temperature for 48 $\mathrm{h}$ to get a white solid (510 mg, 85\% in yield). Calcd: C, 87.01; H, 5.74; N, 7.25. Found: C: $86.20 ; \mathrm{H}, 5.32 ; \mathrm{N}: 7.28$.

P2: P2 was straightforwardly synthesized by the $\mathrm{FeCl}_{3}$ oxidative coupling polymerization of $\mathrm{P} 1$ at room temperature. ${ }^{15}$ The solution of P1 $(200 \mathrm{mg})$ dissolved in $30 \mathrm{~mL}$ anhydrous chloroform was dropwise transferred to a suspension of ferric chloride (1.38 g, $8.49 \mathrm{mmol}$ ) in $20 \mathrm{~mL}$ of anhydrous chloroform. The solution mixture was stirred for $24 \mathrm{~h}$ at room temperature under nitrogen protection, and then $100 \mathrm{~mL}$ of methanol was added to the mixture. The resulting precipitate was collected by filtration and washed with methanol and concentrated hydrochloric acid solution. After extraction in a Soxhlet extractor with methanol for $24 \mathrm{~h}$, and then with THF for another $24 \mathrm{~h}$, the desired polymer was collected as a brown solid and dried in a vacuum oven at $80{ }^{\circ} \mathrm{C}$ overnight (194 mg, 97\% in yield). Calcd: C, 87.01; H, 5.74; N, 7.25. Found: C: 86.37; H: 5.38; N: 6.98.

P3: The solution of $N$-vinyl carbazole $(600 \mathrm{mg}, 3.10 \mathrm{mmol}$ ) dissolved in $30 \mathrm{~mL}$ of anhydrous chloroform was dropwise transferred to a suspension of ferric chloride (4.03 g, 24.84 $\mathrm{mmol}$ ) in $20 \mathrm{~mL}$ of anhydrous chloroform. The solution mixture was stirred for $24 \mathrm{~h}$ at room temperature under nitrogen protection, and then $100 \mathrm{~mL}$ of methanol was added to the mixture. The resulting precipitate was collected by filtration and washed with methanol and concentrated hydrochloric acid solution. After extraction in a Soxhlet extractor with methanol for $24 \mathrm{~h}$, and then with THF for another $24 \mathrm{~h}$, the desired polymer was collected as a dark brown solid and dried in a vacuum oven at $80{ }^{\circ} \mathrm{C}$ overnight (190 mg, 95\% in yield). Calcd: C, 87.01; H, 5.74; N, 7.25. Found: C: 86.93; H: 5.43; N: 6.87.

P4: P3 (200 mg) and AIBN (11 mg, $0.067 \mathrm{mmol}$ ) were dispersed in dry toluene. The solution was heated at $70{ }^{\circ} \mathrm{C}$ under magnetic stirring for $6 \mathrm{~h}$ under a nitrogen atmosphere. The resulting precipitate was collected by filtration and washed with methanol and concentrated hydrochloric acid solution. After extraction in a Soxhlet extractor with methanol for $24 \mathrm{~h}$, and then with THF for another $24 \mathrm{~h}$, the desired polymer was collected as a light brown solid and dried in a vacuum oven at 80 ${ }^{\circ} \mathrm{C}$ overnight (186 mg, 93\% in yield). Calcd: C, 87.01; H, 5.74; N, 7.25. Found: C: 86.48; H: 5.01; N: 6.79. 


\section{Characterization}

The structures of P1 to P4 were confirmed by ${ }^{13} \mathrm{C}$ NMR spectroscopy and Fourier-transform IR (FTIR). P1 was measured by the Bruker Avance III model $600 \mathrm{MHz}$ using $\mathrm{CDCl}_{3}$ as the solvent. ${ }^{13} \mathrm{C} \mathrm{CP} / \mathrm{MAS}$ solid-state NMR measurements were carried out on a Bruker Avance III model $400 \mathrm{MHz}$ NMR spectrometer at a MAS rate of $5 \mathrm{kHz}$ to confirm the structure of P2, P3 and P4. FT-IR spectra were collected in the attenuated total reflection (ATR) mode on a Thermo Nicolet 6700 FT-IR Spectrometer. Thermogravimetric analysis (TGA) was carried out using a SDT Q600 V20.9 Build with a temperature ramp of $10^{\circ} \mathrm{C}$ $\min ^{-1}$ from $20{ }^{\circ} \mathrm{C}$ to $800{ }^{\circ} \mathrm{C}$ under $\mathrm{N}_{2}$ atmosphere. Scanning electron microscopy (SEM) was recorded using a Hitachi S-4800 with acceleration voltage $5.0 \mathrm{kV}$ and working distance $8.9 \mathrm{~mm}$ (P1 and P2) and $8.8 \mathrm{~mm}$ (P3 and P4). Samples were coated with a thin layer of $\mathrm{Au}$ before investigation. Powder X-ray diffractometer (P-XRD) patterns were obtained using a Bruker X-ray diffractometer. Data were collected for $2 \theta$ from $5.00^{\circ}$ to $60.00^{\circ}$ ( $\theta$ is the angle of diffraction). The polymers were degassed at 120 ${ }^{\circ} \mathrm{C}$ for $10 \mathrm{~h}$ under vacuum before analysis to remove residual moisture and other trapped gases. And all the samples were tested on an Autosorb-iQ-MP-VP volumetric adsorption analyzer with the same degassing procedure. The BET surface areas and pore size distributions of P1 to P4 were measured by nitrogen adsorption-desorption at $77 \mathrm{~K}$ in a liquid nitrogen bath. $\mathrm{CO}_{2}$ and $\mathrm{CH}_{4}$ uptake capacities were measured at $273 \mathrm{~K}$ and $298 \mathrm{~K}$ up to 1.1 bar. The reusability was tested by 5 cycles of adsorption and desorption measurements with the same degassing and test procedure above.

\section{Results and discussion}

Four $N$-vinyl carbazole-based polymers were synthesized by different polymerization methods and sequences. Their synthesis procedures are shown in Scheme 1. The free radical polymerization was used in the polymerization of the ethylenic double-bond containing materials. And the $\mathrm{FeCl}_{3}$ oxidative polymerization of carbazole-based building block has been reported to give many kinds of CMPs with impressive performances. The two polymerization methods are both effective ways to synthesize $N$-vinyl carbazole-based polymers.

\section{FTIR and NMR spectra}

The structures of the polymers were characterized by FT-IR and NMR. FTIR spectra (Fig. 1a), in which the strong absorption bands at $721 \mathrm{~cm}^{-1}$ and $744 \mathrm{~cm}^{-1}$ are assigned to the ring deformation of the substituted aromatic structure and $\mathrm{CH}_{2}$ deformation of the vinylidene group, respectively. The bands at $3050 \mathrm{~cm}^{-1}$ and $2930 \mathrm{~cm}^{-1}$ correspond to the stretching vibration of the $\mathrm{C}-\mathrm{H}$ bonds in the aromatic ring and vinylidene group. The obvious absorption bands at $1450 \mathrm{~cm}^{-1}$ and 1330 $\mathrm{cm}^{-1}$ are attributed to the stretching vibration of $\mathrm{C}-\mathrm{H}$ in the aromatic ring and $\mathrm{C}=\mathrm{C}$ in the vinylidene group. The characteristic peak at $1219 \mathrm{~cm}^{-1}$ is the ring vibration of the $N$-vinyl carbazole moiety. It is obvious that the peaks in P2 are broader than those in P1, P3 and P4, which demonstrates the higher
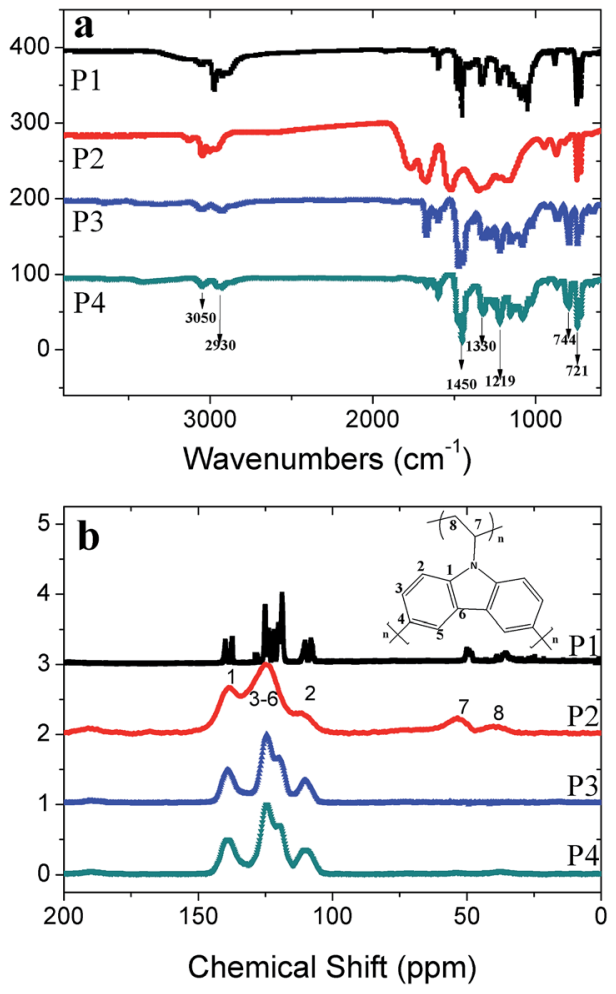

Fig. 1 (a) FTIR spectra for P1 to P4 were collected in ATR mode (the transmittance of P1, P2 and P3 shifted by 100, 200 and 300); (b) solid

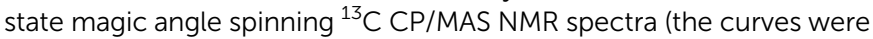
shifted by 1,2 and 3 units after normalization).

degree of polymerization in P1. The peak positions of the obtained polymers are consistent with the infrared PVK spectra reported previously. ${ }^{23}$

It can be seen that in the NMR spectra (Fig. 1b) the four polymers have similar signal positions in the low-field region for the aromatic area. The signal at $139 \mathrm{ppm}$ corresponds to the aryl-carbon binding with the carbazole nitrogen atom, while the broad peaks around $110 \mathrm{ppm}, 120 \mathrm{ppm}$ and $124 \mathrm{ppm}$ are also typical characteristic peaks of $N$-vinyl carbazole. In the NMR spectra of P1 and P2, the peaks at $102 \mathrm{ppm}$ and $129 \mathrm{ppm}$, which are assigned to the double bond of the vinylidene group (Fig. S2 $\dagger$ ), disappear or weaken in the polymers after the free radical polymerization in path 1 . At the same time, the appearance of peaks at $35 \mathrm{ppm}$ and $50 \mathrm{ppm}$ is another proof that part of the vinylidene double bond has transformed into an alkyl group with a single bond. It is obvious that P2 is quite different from P1 as it has a higher degree of polymerization. ${ }^{24}$ P3 and P4, which involve path 2, have similar curve shapes and peak signal positions, although the peaks of P3 are a little sharper than those of $\mathrm{P} 4$. In contrast to path 1 , there are no remarkable peaks at $35 \mathrm{ppm}$ and $50 \mathrm{ppm}$ in the spectra of $\mathrm{P} 3$ and P4. This may be explained in this way: after the first step of polymerization, the materials have obtained the highest phase separation and in the next step just a small amount of the crosslinking reaction takes place on the surface of the interparticles. It is notable that the peaks of P2 are broader than those of its analogues, which could be ascribed to the highest 
degree of polymerization amongst the four polymers. ${ }^{24}$ The results of NMR may show that path 1 is better than path 2, as the high crosslinking degree of monomers is essential to construct porous materials.

\section{TGA, SEM and XRD analysis}

The polymers obtained through path 1 and path 2 were also investigated by thermogravimetric analysis (TGA). The results are shown in Fig. 2a. A small weight loss observed in the initial stages was due to the residual solvents and the adsorbed moisture inside the polymers. ${ }^{25}$ As the temperature increased to $300{ }^{\circ} \mathrm{C}$, P1 started to decompose or evaporate and the sample was totally gone when the temperature rose to $500{ }^{\circ} \mathrm{C}$. The curve of P2 is quite different from P1 curve in path 1 . As to path 2, it is obvious that the curves of $\mathrm{P} 3$ and $\mathrm{P} 4$ are quite similar in shape and turning point. The highest mass loss of $\mathrm{P} 2$ below $410{ }^{\circ} \mathrm{C}$ may be ascribed to the greatest BET surface and largest pore volume available to trap adsorbate. In all cases, P2, P3 and P4 are stable up to $400{ }^{\circ} \mathrm{C}$. Scanning electron microscopy images (Fig. 2b) demonstrate that P1 to $\mathrm{P} 4$ are all aggregation morphologies showing solid sub-micron spheres. In path 1, P1 and $\mathrm{P} 2$ are composed of particles with different sizes, whereas, in the case of path 2, one can clearly see that the morphology of P3 is different from that of P4. P-XRD (Fig. S3†) shows that P1 has two diffraction peaks at around $7.5^{\circ}$ and $23.5^{\circ}$, both characteristic peaks of PVK, in agreement with previous reports. ${ }^{26}$ The broad peak from $15^{\circ}$ to $25^{\circ}$ in $\mathrm{P} 2$ to $\mathrm{P} 4$ indicates that most of the $N$-vinyl carbazole was polymerized to form amorphous random materials.

\section{Pore properties analysis}

Nitrogen adsorption-desorption isotherms were measured to analyze the porous properties of microporous polymers. ${ }^{19,27,28}$ The surface areas of P1 to P4 calculated by the BrunauerEmmett-Teller (BET) method in the relative pressure $\left(P / P_{0}\right)$ range from 0.05 to 0.20 are $68.65 \mathrm{~m}^{2} \mathrm{~g}^{-1}, 878.46 \mathrm{~m}^{2} \mathrm{~g}^{-1}, 621.18$ $\mathrm{m}^{2} \mathrm{~g}^{-1}$ and $660.62 \mathrm{~m}^{2} \mathrm{~g}^{-1}$, respectively (Fig. 3b). The pore size distributions were calculated from the adsorption branches of the isotherms using the quenched solid density functional theory (QSDFT). As can be clearly seen, the pore width of the four polymers is $3.94 \mathrm{~nm}, 0.54 \mathrm{~nm}, 1.13 \mathrm{~nm}$ and $1.17 \mathrm{~nm}$, respectively. Detailed information on the porosity structural parameters of $\mathrm{P} 1$ to $\mathrm{P} 4$ is shown in Table 1.

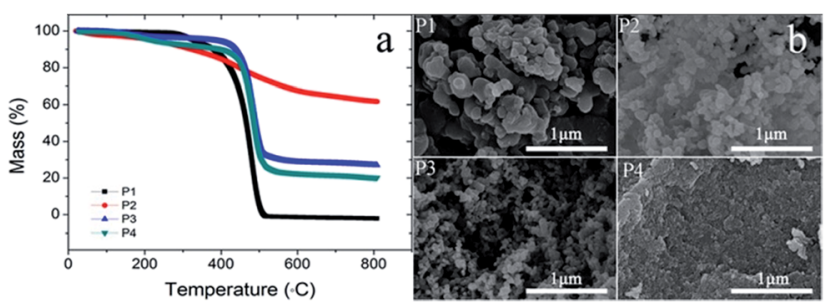

Fig. 2 (a) Thermogravimetric analysis of P1 to P4; (b) field-emission scanning electron microscopy images of P1 to P4 (scale bar: $1 \mu \mathrm{m}$ ).

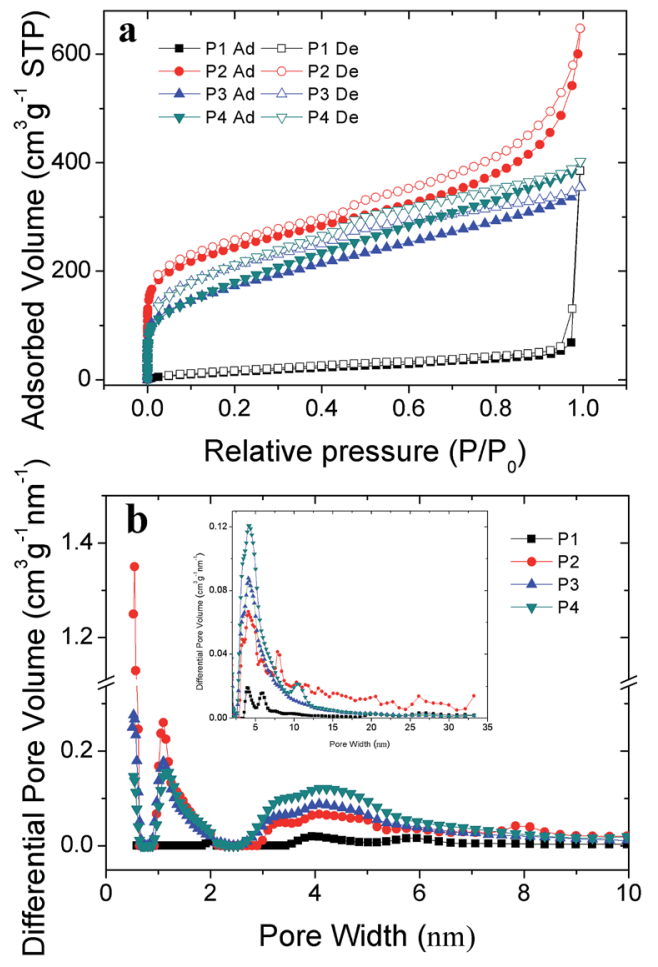

Fig. 3 a) Nitrogen adsorption-desorption isotherms of polymers measured at $77 \mathrm{~K}$; (b) pore size distributions calculated using quenched solid density functional theory; the inset is the mesopore size distribution ranging from 2 to $35 \mathrm{~nm}$.

P1: The adsorption-desorption isotherm of P1 demonstrates that it is a mesoporous material, as there is little increase at a relatively low pressure and a steep gas uptake at a relatively high pressure $\left(P / P_{0}=0.9-1.0\right)$. The pore size distribution of $\mathrm{P} 1$ is another proof of its mesoporous nature, as its pore width is mainly $3.94 \mathrm{~nm}$. P1 is a linear polymer which can be classified as polymer of intrinsic microporous (PIMs) analogues because the $N$-vinyl carbazole is not rigid enough to construct a microporous architecture by connecting only the ethylenic double bonds together.

As an ultramicropore conjugated polymer, among the four polymers P2 has the largest BET surface areas $\left(878.46 \mathrm{~m}^{2} \mathrm{~g}^{-1}\right)$ and narrowest pore size distribution centered at $0.54 \mathrm{~nm}$. The isotherm of P2 shows a steep increase at a relatively low pressure, a slow increase at a relatively intermediate pressure, and a

Table 1 Porosity structural parameters of P1 to P4

\begin{tabular}{llclll}
\hline Polymer & $\begin{array}{l}S_{\mathrm{BET}} \\
{\left[\mathrm{m}^{2} \mathrm{~g}^{-1}\right]^{a}}\end{array}$ & $\begin{array}{l}S_{\text {Lang }} \\
{\left[\mathrm{m}^{2} \mathrm{~g}^{-1}\right]}\end{array}$ & $\begin{array}{l}V_{\text {O.1 }} \\
{\left[\mathrm{cm}^{3} \mathrm{~g}^{-1}\right]^{b}}\end{array}$ & $\begin{array}{l}V_{\text {total }} \\
{\left[\mathrm{cm}^{3} \mathrm{~g}^{-1}\right]^{c}}\end{array}$ & $\begin{array}{l}\text { Width } \\
{[\mathrm{nm}]^{d}}\end{array}$ \\
\hline P1 & 69 & 137 & 0.10 & 0.60 & 3.94 \\
P2 & 878 & 1105 & 0.25 & 1.00 & 0.54 \\
P3 & 621 & 846 & 0.16 & 0.55 & 1.13 \\
P4 & 661 & 928 & 0.24 & 0.66 & 1.17
\end{tabular}

${ }^{a}$ Surface area calculated from the $\mathrm{N}_{2}$ isotherm. ${ }^{b}$ The micropore volume at $P / P_{0}=0.10{ }^{c}$ Total pore volume at $P / P_{0}=0.99 .{ }^{d}$ Dominant pore size calculated by quenched solid density functional theory (QSDFT). 
sharp gas uptake at a relatively high pressure. The high adsorption at low pressure can be interpreted as surface adsorption and micropore filling. And the gradual increase at relatively high pressure may be due to the meso/ macrostructures and interparticular void in the polymer. The post-polymerization of $\mathrm{P} 1$ in the second step of path 1 prompts the 3,6-position in the carbazole group to connect successfully, and the obtained $\mathrm{P} 2$ shows a narrower pore distribution with a great improvement in the BET surface areas and pore volume, which are beneficial for gas storage and separation. It can thus be seen that P2 as a post-polymer of P1 is far better than its initial parent polymer in all aspects.

It was found that P3 and P4 are both typical CMPs, possessing similar nitrogen adsorption and desorption isotherms. P3 and P4 share a nearly equivalent BET surface areas of 621.18 $\mathrm{m}^{2} \mathrm{~g}^{-1}$ and $660.62 \mathrm{~m}^{2} \mathrm{~g}^{-1}$, respectively. Furthermore, the two polymers exhibit the same dominant pore width centered at $1.13 \mathrm{~nm}$ and $1.17 \mathrm{~nm}$. P4 shows a small increase of surface area and pore volume when compared with $\mathrm{P} 3$, which can be attributed to a little crosslinking reaction taking place on the surface of interparticles and the swelling effect in the second step of path 2. The accordant pore-size distributions of P3 and P4 demonstrate that the second step of path 2 does not change the pore width, which is determined by the stiff backbone. It also illustrates that the reaction happened on the surface of the interparticles only, but not internally in the P3.

Taking path 1 and path 2 in comparison, one can easily conclude that the former is superior to the latter, as P2 is much better than P4 in its porous properties. Path 1, which is cost effective, high yielding and pollution-free, should be regarded as the optimum choice to prepare the $N$-vinyl carbazole-based porous materials.

\section{Gas uptake}

Carbon dioxide adsorption. Nowadays, excessive $\mathrm{CO}_{2}$ emissions have caused a series of environmental and geological problems. The capture of $\mathrm{CO}_{2}$, relying on physical adsorption, is an emerging alternative solution to mitigate environmental damage. Compared with the traditional amine-based process, which has high energy demands, ${ }^{29}$ the prominent advantage of the dry solid sorbent is the low regeneration energy of $\mathrm{CO}_{2}$ capture. MOPs are of great interest for $\mathrm{CO}_{2}$ capture because they combine a porous nature with organic functionalities which can be modified for $\mathrm{CO}_{2}$ capture. In this work, the four carbazolebased polymers are nitrogen-containing and show high pore volumes, which are beneficial for $\mathrm{CO}_{2}$ capture. Here, the four polymers are also investigated as solid sorbents for $\mathrm{CO}_{2}$ uptake. The $\mathrm{CO}_{2}$ isotherms of the four polymers are shown in Fig. 4a. The overlapped adsorption and desorption branches demonstrate there were no elastic deformations in the course of $\mathrm{CO}_{2}$ sorption. The $\mathrm{CO}_{2}$ uptakes of the polymers at $273 \mathrm{~K} / 1.0$ bar are $2.26 \mathrm{wt} \%, 12.95 \mathrm{wt} \%, 8.62 \mathrm{wt} \%$ and $9.19 \mathrm{wt} \%$ from $\mathrm{P} 1$ to $\mathrm{P} 4$, respectively. P2, P3 and $\mathrm{P} 4$ exhibit $\mathrm{CO}_{2}$ uptakes higher than or comparable to the reported porous materials, such as TFM-1 $\left(S_{\mathrm{BET}}=738 \mathrm{~m}^{2} \mathrm{~g}^{-1}, 7.6 \mathrm{wt} \%\right),{ }^{30} \mathrm{COF}-102\left(S_{\mathrm{BET}}=3620 \mathrm{~m}^{2} \mathrm{~g}^{-1}\right.$, $\left.1.16 \mathrm{mmol} \mathrm{g}{ }^{-1}=5.1 \mathrm{wt} \%\right),{ }^{31}$ SPOP3 $\left(S_{\text {BET }}=967 \mathrm{~m}^{2} \mathrm{~g}^{-1}, 11.6\right.$
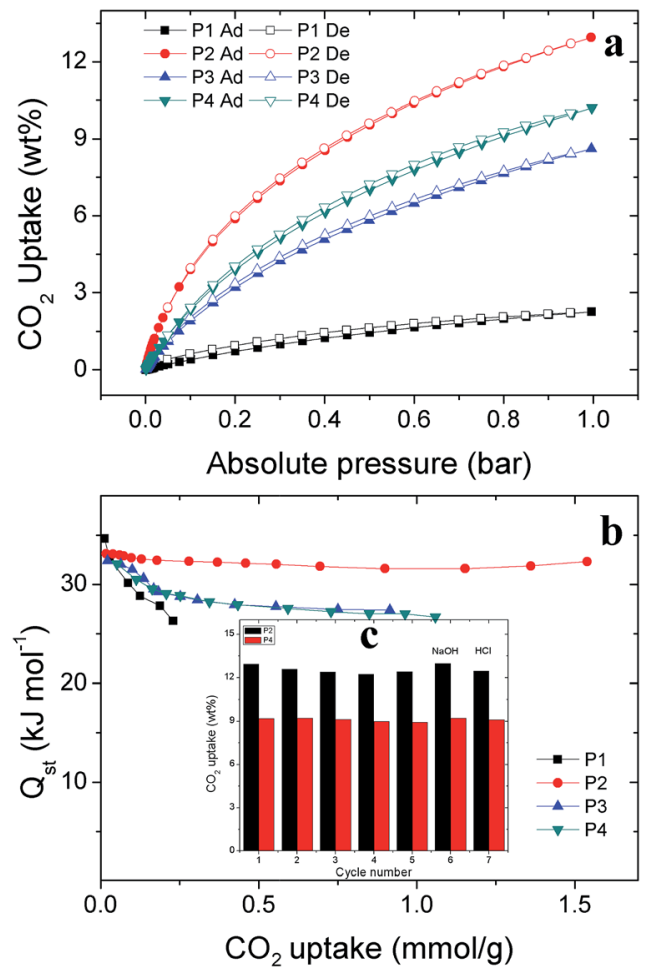

Fig. 4 (a) $\mathrm{CO}_{2}$ adsorption-desorption properties of the polymers at $273 \mathrm{~K}$; (b) $\mathrm{CO}_{2}$ isosteric enthalpies with the amount adsorbed (calculated by $\mathrm{CO}_{2}$ adsorption isotherms of the polymers at $273 \mathrm{~K}$ and $298 \mathrm{~K}$ ); (c) reusability of P2 and P4 in five cycles and stability of P2 and P4 after treatment with $\mathrm{NaOH}(6 \mathrm{M})$ and $\mathrm{HCl}(6 \mathrm{M})$ for $1 \mathrm{~d}$.

wt $\%)^{32}$ and ZIF-69 $\left(S_{\text {BET }}=1220 \mathrm{~m}^{2} \mathrm{~g}^{-1}, 13.5 \mathrm{wt} \%\right){ }^{33}$ The high uptake of $\mathrm{CO}_{2}$ can be ascribed to the $\mathrm{N}$-containing structure in the carbazole unit. It is known that $\mathrm{CO}_{2}$ is an acidic adsorbate with a quadrupole moment, and the alkaline nitrogen sites on the surface can facilitate the dipole-quadrupole interaction and acid/base interaction between the polymer and $\mathrm{CO}_{2}$ molecule, which can greatly strengthen the uptake of $\mathrm{CO}_{2}$. To determine the strength of the interaction between molecular $\mathrm{CO}_{2}$ and polymers, the isosteric heats of adsorption were calculated from the $\mathrm{CO}_{2}$ isotherms measured at $273 \mathrm{~K}$ and $298 \mathrm{~K}$ (Fig. S4 $\dagger$ ). The heats of adsorption for $\mathrm{CO}_{2}$ are in the range of $25.60 \mathrm{~kJ} \mathrm{~mol}^{-1}$ to $34.26 \mathrm{~kJ} \mathrm{~mol}^{-1}$ (Fig. 4b), which are higher than or comparable to the values of other reported porous materials (TFM-1, $27.8 \mathrm{~kJ}$ $\mathrm{mol}^{-1}$; CMP-1-COOH, $\left.32.6 \mathrm{~kJ} \mathrm{~mol}^{-1}\right){ }^{30,34} \mathrm{P} 2$, which shows the best porous properties, is quite outstanding, because the enthalpy of adsorption is about $32 \mathrm{~kJ} \mathrm{~mol}^{-1}$, without obvious reduction as the loading increases. P2 to P4 exhibited a comparatively high $\mathrm{CO}_{2}$ adsorption and enthalpy, indicating that the polymers are good $\mathrm{CO}_{2}$-capture materials. ${ }^{35,36}$ It is worth noting that the heats of strong physisorption are less than the energy needed for chemical adsorption, which makes these polymers desirable for facile $\mathrm{CO}_{2}$ release in practical applications.

To further investigate the reusability and stability of polymers, P2 and P4 were chosen to go through five cycles and acid/ base treatment because of their large BET surface areas and pore volume. As shown in Fig. 4c, the $\mathrm{CO}_{2}$ uptake of P2 and P4 
does not decline after 5 cycles. In addition, the polymers are stable, even when treated with $6 \mathrm{M} \mathrm{NaOH}$ and $6 \mathrm{M} \mathrm{HCl}$ solutions. These results demonstrate that the pore structures of P2 and $\mathrm{P} 4$ are robust and that their performance will not weaken, even under rigorous conditions.

Hydrogen storage. It has been reported that materials with low density and high porosity are promising candidates for gasstorage applications. MOPs are good candidates for gas adsorption and storage, due to their high specific surface area, narrow pore size distribution and low framework density. Hydrogen as a outstanding fuel is an alternative clean energy source owing to its zero pollution and high energy density. MOPs have exhibited potential in hydrogen storage for their high uptake at $77 \mathrm{~K}$ and 1 bar, such as CPOP- $1\left(S_{\mathrm{BET}}=2220 \mathrm{~m}^{2}\right.$ $\left.\mathrm{g}^{-1}, 2.80 \mathrm{wt} \%\right),{ }^{18}$ BILP-1 $\left(S_{\mathrm{BET}}=1172 \mathrm{~m}^{2} \mathrm{~g}^{-1}, 1.90 \mathrm{wt} \%\right)^{37}$ and PPN-3 $\left(S_{\text {BET }}=2840 \mathrm{~m}^{2} \mathrm{~g}^{-1}, 1.58 \mathrm{wt} \%\right){ }^{38}$ The hydrogen uptakes of polymers (Fig. $5 \mathrm{a}$ ) at $77 \mathrm{~K} / 1.0 \mathrm{bar}$ are $0.37 \mathrm{wt} \%, 1.36 \mathrm{wt} \%, 1.15$ $\mathrm{wt} \%$ and $1.23 \mathrm{wt} \%$, respectively. The trends of hydrogen uptake coincide with those of the BET surface areas and pore volumes of the polymers, as the dominant interaction of hydrogen with surface is the Van der Waals attraction. In contrast to microporous metal-organic frameworks (MOFs), only physical absorption occurs for P1 to P4 as there are no metal sites which initiate the propagation of hydrogen adsorption across the whole framework via a cooperative mechanism. ${ }^{39}$ The hydrogenstorage capacity of $\mathrm{P} 2$ is among the highest reported for pore materials, although the BET surface area is only $878 \mathrm{~m}^{2} \mathrm{~g}^{-1}$. In addition, the cycle tests indicate the stability of P2 and P4, shown in Fig. 5c. The performance of P2 and P4 did not change much and the deviation could be caused by test error. The stable performance of these polymers can ensure their hydrogen capacity after several cycles of use. However, the research is still in the test phase, and much work needs to be done to apply these materials in hydrogen storage.
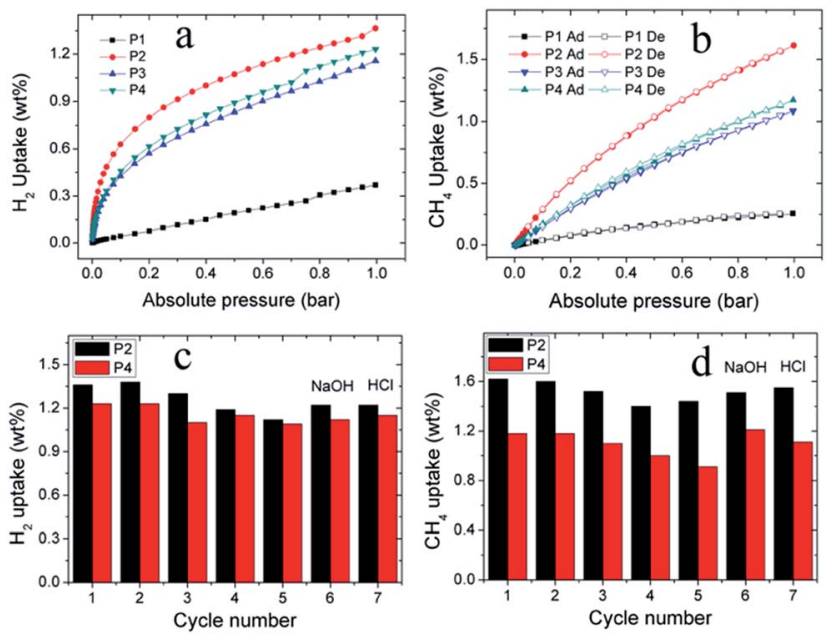

Fig. 5 (a) $\mathrm{H}_{2}$ adsorption at $77 \mathrm{~K}$; (b) $\mathrm{CH}_{4}$ adsorption at $273 \mathrm{~K}$; (c) reusability in uptake of $\mathrm{H}_{2}$ by $\mathrm{P} 2$ and $\mathrm{P} 4$ in five cycles and stability of $\mathrm{P} 2$ and $\mathrm{P} 4$ after treatment with $\mathrm{NaOH}(6 \mathrm{M})$ and $\mathrm{HCl}(6 \mathrm{M})$ for 1 day. (d) reusability in uptake of $\mathrm{CH}_{4}$ by $\mathrm{P} 2$ and $\mathrm{P} 4$ in five cycles and stability of $\mathrm{P} 2$ and $\mathrm{P} 4$ after treatment with $\mathrm{NaOH}(6 \mathrm{M})$ and $\mathrm{HCl}(6 \mathrm{M})$ for 1 day.

\section{Methane uptake}

Although the combustion of methane produces $\mathrm{CO}_{2}$, it still has environmental advantages as a transitional fuel source with respect to other fossil fuels, due to its high ratio of hydrogen to carbon. Furthermore, it is available in large quantities, like combustible ice. Storing methane via adsorption on porous materials is the most promising near-term route because it allows operation at reasonable pressures (1-300 bar) and temperatures (7-298 K) and does not require extra energy input for conversion into higher hydrocarbons or methanol. Adsorption in nanoporous materials such as MOPs is very efficient and promising, because it can obtain methane densities comparable to those of the compressed phase at a much lower pressure and ambient temperature in lightweight carriers. ${ }^{40}$ The four polymers with a low framework density and large pore volume are alternatives in methane storage. The uptake capacities for methane of the prepared polymers were measured at $273 \mathrm{~K}$ from zero to 1.0 bar. As shown in Fig. 5b, methane uptakes of P1 to P4 are $0.26 \mathrm{wt} \%, 1.62 \mathrm{wt} \%, 1.08 \mathrm{wt} \%$ and 1.18 wt $\%$ at $273 \mathrm{~K} / 1.0$ bar, respectively. One can observe that methane-uptake capacities increases corresponding to the BET surface areas and total pore volume, as the order of the methane uptake ability is $\mathrm{P} 2>\mathrm{P} 4>\mathrm{P} 3>\mathrm{P} 1$.

The dominant interaction of methane with the surface is the Van der Waals attraction. Goddard and coworkers have found that $\mathrm{CH}_{4}$ adsorption in polymers can occur with the coexistence of layer formation and pore filling. ${ }^{41}$ Porous materials show potential applications in the purification of clean-burning natural gas, ${ }^{36}$ as the existence of $\mathrm{CO}_{2}$ in natural gas can reduce the energy density of fuel. A promising material for practical application requires not only a high $\mathrm{CO}_{2}$ uptake but also excellent $\mathrm{CO}_{2}$ selectivity. The $\mathrm{CO}_{2} / \mathrm{CH}_{4}$ selectivities of P1 to $\mathrm{P} 4$ are calculated using the initial slopes at low pressure in the Henry's law region. The calculations show that the $\mathrm{CO}_{2} / \mathrm{CH}_{4}$ selectivities of $\mathrm{P} 1$ to $\mathrm{P} 4$ are 4.34, 6.9, 5.7 and 5.71, respectively. As the four polymers are polymerized from the same monomers, the differences in selectivity may be caused mainly by the pore structure.

\section{Conclusions}

We report two facile strategies with alternative polymerization sequences to prepare ( $N$-vinyl carbazole)-based porous polymers for gas storage. The two strategies can synthesize polymers with high-yielding, cost-effective and full utilization of the two different active sites of the $N$-vinyl carbazole unit by free radical polymerization and $\mathrm{FeCl}_{3}$ oxidative polymerization. The BET surface areas of the obtained materials P1 to P4 show a large range from $68.65 \mathrm{~m}^{2} \mathrm{~g}^{-1}$ to $878.46 \mathrm{~m}^{2} \mathrm{~g}^{-1}$. In path 1 , in contrast to the linear PIMs analogue P1 with a dominant pore size of 3.94 $\mathrm{nm}, \mathrm{P} 2$ is an interpenetrating ultramicropore conjugated polymer (dominant pore size $0.54 \mathrm{~nm}$ ). In path 2, P4 shows slightly higher BET surface areas than, and similar pore width distribution to, P3, indicating that little crosslinking reaction occurs at the next polymerization stage. The $\mathrm{CO}_{2}, \mathrm{H}_{2}$, and $\mathrm{CH}_{4}$ adsorption isothermals show that P2 has the highest gas-uptake capacity 
among the four polymers (for $\mathrm{CO}_{2}$ it is $12.95 \mathrm{wt} \%$ at $273 \mathrm{~K} / 1.0$ bar, for $\mathrm{H}_{2}$ it is $1.36 \mathrm{wt} \%$ at $77 \mathrm{~K} / 1.0$ bar and for $\mathrm{CH}_{4}$ it is 1.62 $\mathrm{wt} \%$ at $273 \mathrm{~K} / 1.0 \mathrm{bar}$ ), which is comparable to the highest reported for CMPs under similar conditions. The stabilities of P2 and $\mathrm{P} 4$ were investigated. It turns out they are quite stable after 5 cycles. The gas uptake results indicate that path 1 , which is cost effective, high-yielding and pollution free, can be regarded as the optimum way to prepare poly( $N$-vinyl carbazole) CMPs. This work clearly reveals that selection of the appropriate monomer and the right polymerization strategies are crucial considerations for materials with excellent gas-uptake capacities.

\section{Acknowledgements}

This work was supported by National Natural Science Foundation of China (21274161, 51173199, 51303197), the Ministry of Science and Technology of China (2014CB643501, 2010DFA52310), Shandong Provincial Natural Science Foundation (ZR2011BZ007), and Qingdao Municipal Science and Technology Program (11-2-4-22-hz).

\section{Notes and references}

1 R. Dawson, A. I. Cooper and D. J. Adams, Prog. Polym. Sci., 2012, 37, 530-563.

2 N. B. McKeown and P. M. Budd, Macromolecules, 2010, 43, 5163-5176.

3 W. Lu, D. Yuan, J. Sculley, D. Zhao, R. Krishna and H.-C. Zhou, J. Am. Chem. Soc., 2011, 133, 18126-18129.

4 T. Ben, H. Ren, S. Ma, D. Cao, J. Lan, X. Jing, W. Wang, J. Xu, F. Deng and J. M. Simmons, Angew. Chem., Int. Ed., 2009, 121, 9457-9460.

5 P. Kaur, J. T. Hupp and S. T. Nguyen, ACS Catal., 2011, 1, 819-835.

6 L. Chen, Y. Yang and D. Jiang, J. Am. Chem. Soc., 2010, 132, 9138-9143.

7 A. P. Cote, A. I. Benin, N. W. Ockwig, M. O'Keeffe, A. J. Matzger and O. M. Yaghi, Science, 2005, 310, 1166-1170.

8 J. Xia, S. Yuan, Z. Wang, S. Kirklin, B. Dorney, D.-J. Liu and L. Yu, Macromolecules, 2010, 43, 3325-3330.

9 N. B. McKeown and P. M. Budd, Chem. Soc. Rev., 2006, 35, 675-683.

10 A. I. Cooper, Adv. Mater., 2009, 21, 1291-1295.

11 M. Tsyurupa and V. Davankov, React. Funct. Polym., 2006, 66, 768-779.

12 J. H. Choi, K. M. Choi, H. J. Jeon, Y. J. Choi, Y. Lee and J. K. Kang, Macromolecules, 2010, 43, 5508-5511.

13 J. X. Jiang, F. Su, A. Trewin, C. D. Wood, N. L. Campbell, H. Niu, C. Dickinson, A. Y. Ganin, M. J. Rosseinsky and Y. Z. Khimyak, Angew. Chem., Int. Ed., 2007, 46, 8574-8578.

14 Y. Kou, Y. Xu, Z. Guo and D. Jiang, Angew. Chem., 2011, 123, 8912-8916.

15 S. Qiao, Z. Du and R. Yang, J. Mater. Chem. A, 2014, 2, 18771885.

16 J. Weber and A. Thomas, J. Am. Chem. Soc., 2008, 130, 63346335.
17 Y. Xu, L. Chen, Z. Guo, A. Nagai and D. Jiang, J. Am. Chem. Soc., 2011, 133, 17622-17625.

18 Q. Chen, M. Luo, P. Hammershøj, D. Zhou, Y. Han, B. W. Laursen, C.-G. Yan and B.-H. Han, J. Am. Chem. Soc., 2012, 134, 6084-6087.

19 S. Qiao, Z. Du, C. Yang, Y. Zhou, D. Zhu, J. Wang, X. Chen and R. Yang, Polymer, 2014, 55, 1177-1182.

20 C. Gu, N. Huang, J. Gao, F. Xu, Y. Xu and D. Jiang, Angew. Chem., Int. Ed., 2014, 53, 4850-4855.

21 N. Tsutsumi, K. Kinashi, A. Nonomura and W. Sakai, Materials, 2012, 5, 1477-1486.

22 T. H. Kuo, C. J. Weng, C. L. Chen, Y. L. Chen, C. H. Chang and J. M. Yeh, Polym. Compos., 2012, 33, 275-281.

23 K. I. Kim, C. Basavaraja and D. S. Huh, Bull. Korean Chem. Soc., 2013, 34, 1391-1396.

24 D. S. Das, D. P. Chatterjee and A. K. Nandi, J. Mater. Chem. A, 2014, 2, 12031-12042.

25 Y. Zhu, H. Long and W. Zhang, Chem. Mater., 2013, 25, 16301635.

26 A. T. Gokceoren and C. Erbil, Polym. Compos., 2013, 34, 1986-1998.

27 Q. Zhang, Y. Yang and S. Zhang, Chem.-Eur. J., 2013, 19, 10024-10029.

28 Z. Wang, B. Zhang, H. Yu, G. Li and Y. Bao, Soft Matter, 2011, 7, 5723-5730.

29 A. Samanta, A. Zhao, G. K. Shimizu, P. Sarkar and R. Gupta, Ind. Eng. Chem. Res., 2011, 51, 1438-1463.

30 X. Zhu, C. Tian, S. M. Mahurin, S.-H. Chai, C. Wang, S. Brown, G. M. Veith, H. Luo, H. Liu and S. Dai, J. Am. Chem. Soc., 2012, 134, 10478-10484.

31 H. Furukawa and O. M. Yaghi, J. Am. Chem. Soc., 2009, 131, 8875-8883.

32 Q. Chen, J.-X. Wang, Q. Wang, N. Bian, Z.-H. Li, C.-G. Yan and B.-H. Han, Macromolecules, 2011, 44, 7987-7993.

33 R. Banerjee, A. Phan, B. Wang, C. Knobler, H. Furukawa, M. O'Keeffe and O. M. Yaghi, Science, 2008, 319, 939-943.

34 R. Dawson, D. J. Adams and A. I. Cooper, Chem. Sci., 2011, 2, 1173-1177.

35 Y. Xie, T.-T. Wang, X.-H. Liu, K. Zou and W.-Q. Deng, Nat. Commun., 2013, 4, 1-7.

36 W.-C. Song, X.-K. Xu, Q. Chen, Z.-Z. Zhuang and X.-H. Bu, Polym. Chem., 2013, 4, 4690-4696.

37 M. G. Rabbani and H. M. El-Kaderi, Chem. Mater., 2011, 23, 1650-1653.

38 W. Lu, D. Yuan, D. Zhao, C. I. Schilling, O. Plietzsch, T. Muller, S. Bräse, J. Guenther, J. Blümel and R. Krishna, Chem. Mater., 2010, 22, 5964-5972.

39 T. B. Lee, D. Kim, D. H. Jung, S. B. Choi, J. H. Yoon, J. Kim, K. Choi and S.-H. Choi, Catal. Today, 2007, 120, 330-335.

40 X. Zhu and Y.-P. Zhao, J. Phys. Chem. C, 2014, 118, 1773717744.

41 J. L. Mendoza-Cortes, S. S. Han, H. Furukawa, O. M. Yaghi and W. A. Goddard III, J. Phys. Chem. A, 2010, 114, 1082410833. 